\title{
Difference in Transcranial Doppler Velocity and Patient Age between Proximal and Distal Middle Cerebral Artery Vasospasms after Aneurysmal Subarachnoid Hemorrhage
}

\author{
Misaki Kohama $^{a}$ Shinichiro Sugiyama ${ }^{a}$ Kenichi Sato $^{b}$ Hidenori Endo $^{c}$ \\ Kuniyasu Niizumad ${ }^{d}$ Toshiki Endo $^{c}$ Makoto Ohta ${ }^{e}$ Yasushi Matsumoto $^{b}$ \\ Miki Fujimurad Teiji Tominagad $^{d}$ \\ Departments of a Neuroanesthesia, ${ }^{b}$ Neuroendovascular Therapy and ${ }^{c}$ Neurosurgery, \\ Kohnan Hospital, d Department of Neurosurgery, Tohoku University Graduate School of \\ Medicine, and Institute of Fluid Science, Tohoku University, Sendai, Japan
}

Key Words

Subarachnoid hemorrhage · Cerebral vasospasm · Transcranial Doppler · Ultrasonography

\section{Abstract}

Background: Transcranial Doppler (TCD) is used to monitor cerebral vasospasm after subarachnoid hemorrhage (SAH), but its diagnostic ability is reported to be limited. Therefore, the purpose of this study was to investigate the relationship between the diagnosability of TCD and the localization of the vasospasm. Methods: This retrospective study included 20 patients who presented with symptomatic vasospasm after SAH. All 20 patients underwent daily TCD examinations and cerebral angiography after the onset of delayed cerebral ischemia. We defined positive findings on TCD as a maximum flow velocity $>200 \mathrm{~cm} / \mathrm{s}$ or as a mean flow velocity $>120 \mathrm{~cm} / \mathrm{s}$ at the horizontal part of the middle cerebral artery (MCA). We also examined the site of vasospasm on cerebral angiography. Results: Fourteen patients had true-positive findings on TCD examination, and cerebral angiography showed diffuse vasospasm involving the horizontal segment of the MCA. However, 6 patients had false-negative findings on TCD examination, and cerebral angiography showed vasospasm localized at the distal part of the MCA (the insular and/or cortical segments). The patients with proximal vasospasm were significantly younger than those with distal vasospasm. Blood flow velocity at initial TCD and the increase in velocity at the onset of vasospasm were lower and smaller, respectively, in the distal vasospasm group. Conclusions: In patients with cerebral vasospasm localized at the distal part of the MCA, flow velocity at the horizontal segment of the MCA did not increase to the level we defined as positive. To avoid such false negatives, a slight increase in velocity on TCD should be considered as positive in distal vasospasm cases, especially in older patients. 

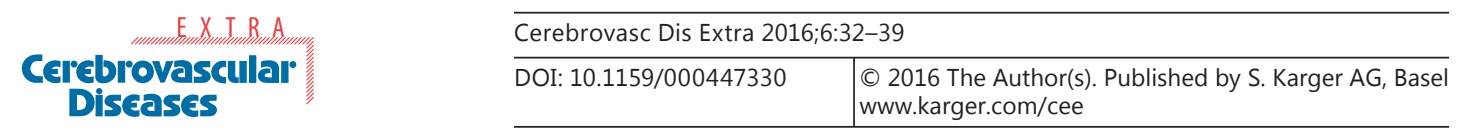

Kohama et al.: Difference in TCD Velocity and Patient Age between Proximal and Distal MCA Vasospasms after Aneurysmal SAH

\section{Background}

Symptomatic cerebral vasospasm is one of the major problems after subarachnoid hemorrhage (SAH) [1]. It causes delayed cerebral ischemia and persistent neurological deficit in $20-40 \%$ of patients with SAH [1-4]. Therefore, early diagnosis followed by therapeutic intervention is needed to improve the outcome of this disease.

Almost 30 years ago, transcranial Doppler (TCD) was introduced for noninvasive monitoring of cerebral vasospasms [5]. It provides information about the blood flow of basal cerebral arteries, and a cerebral vasospasm is diagnosed by the increase in blood flow velocity at the examined vessels, based on the principle of fluid dynamics that the velocity in a tube is inversely related to the area of the lumen. Because TCD examination can be performed daily at bedside, it is utilized for diagnosing cerebral vasospasm at many neurosurgical units [6, 7]. In addition, a sophisticated TCD device, transcranial color flow image (TCCFI), has recently emerged. In the revised American Heart Association guidelines for the management of SAH, TCD examination is recommended to monitor for the development of cerebral vasospasm (class IIa; level of evidence B) [8].

However, the usefulness of TCD/TCCFI is controversial. A recent systemic review of seven trials examining the correlation between the results of TCD and cerebral angiography suggested that the diagnostic ability of TCD is limited [6]. The usefulness of TCD was confirmed only for the middle cerebral artery (MCA) and not for the other cerebral arteries [6]. For cerebral vasospasm of the MCA, the specificity and positive predictive value of TCD are both high, but the sensitivity and negative predictive value are both low [6]. The latter means that TCD provides negative findings even when cerebral angiography is positive, and thus we cannot rule out cerebral vasospasm even if TCD provides negative findings.

A systematic review showed that the limited ability of TCD for diagnosing cerebral vasospasm is due to the high false-negative value [6]. Although operator inexperience or technical failures were reported to be responsible for false-negatives on TCD in cerebral arteries, except for the MCA, it is unclear why the TCD examination provides false-negative information even in the MCA.

From a rheological viewpoint, we speculated that the false negatives on TCD examination are caused by localization of the vasospasm. According to fluid dynamics, the flow velocity does not increase at the artery proximal to the stenotic legion, unless the flow volume increases. Our hypothesis was that flow velocity at the horizontal segment of the MCA (M1) does not increase unless M1 is involved in the vasospasm.

This retrospective study included patients diagnosed with symptomatic vasospasm after SAH by cerebral angiography. We investigated whether the cause of false negatives on TCD examination was related to the localization of the vasospasm. In addition, we demonstrate the correlation between the localization of vasospasm and the age of patients.

\section{Methods}

We retrospectively investigated the clinical and radiological information of 702 patients with aneurysmal SAH admitted to our hospital from May 2008 to April 2015. The treatment strategy for aneurysmal SAH at our hospital is described in supplemental data (for all online suppl. material, see www.karger.com/doi/10.1159/000447330). TCCFI examination of both MCAs was performed daily during the patient's admission at an intensive care unit using the TCCFI device (Logiq P5; GE Healthcare, Tokyo, Japan). The maximum and mean flow velocity of the M1 on TCCFI examination was recorded. 


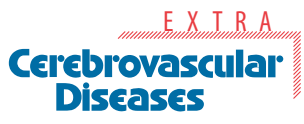

Table 1. Patient characteristics for groups A and B

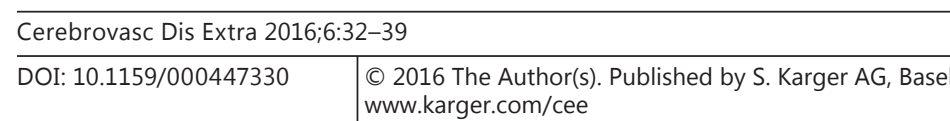

Kohama et al.: Difference in TCD Velocity and Patient Age between Proximal and Distal MCA Vasospasms after Aneurysmal SAH

\begin{tabular}{|c|c|c|}
\hline & $\begin{array}{l}\text { Group A } \\
(\%)\end{array}$ & $\begin{array}{l}\text { Group B } \\
(\%)\end{array}$ \\
\hline Patients, $\mathrm{n}$ & 14 & 6 \\
\hline Age* & $48.4 \pm 10.3$ & $73.7 \pm 8.7$ \\
\hline Female & $7(50.0)$ & $4(66.7)$ \\
\hline \multicolumn{3}{|l|}{ Hunt and Kosnik grade } \\
\hline 1 & 0 & $1(16.7)$ \\
\hline 2 & $5(35.7)$ & 0 \\
\hline 3 & $4(28.6)$ & $4(66.7)$ \\
\hline 4 & $5(35.7)$ & $1(16.7)$ \\
\hline 5 & 0 & 0 \\
\hline \multicolumn{3}{|l|}{ Location of aneurysm } \\
\hline ICA & $4(28.6)$ & $3(50.0)$ \\
\hline MCA & $3(21.4)$ & $2(33.3)$ \\
\hline ACA, AcomA & $3(21.4)$ & 1 (16.7) \\
\hline VA, BA & $4(28.6)$ & 0 \\
\hline \multicolumn{3}{|l|}{ Treatment } \\
\hline Microsurgical clipping & $4(28.6)$ & $4(66.7)$ \\
\hline Neuroendovascular treatment & $10(71.4)$ & $2(33.3)$ \\
\hline \multicolumn{3}{|l|}{ Outcome } \\
\hline Good (modified Rankin scale $0-2$ ) & $10(71.4)$ & $3(50.0)$ \\
\hline Poor (modified Rankin scale $\geq 3$ ) & $4(28.6)$ & $3(50.0)$ \\
\hline
\end{tabular}

Inclusion criteria of this study were as follows: (1) treatment consisted of microsurgical clipping or endovascular coiling, (2) delayed cerebral ischemia, (3) cerebral angiography available to examine the site of vasospasm at the MCA, (4) available for daily TCCFI examinations, and (5) single-photon emission computed tomography (SPECT) available to examine cerebral blood flow.

To assess the localization of MCA vasospasm with angiography, we adopted the conventional neuroradiological definition of the MCA by Fischer [9]. The MCA is divided into three segments: the horizontal segment (M1), the insular segment (M2), and the cortical segment (M3). We classified cerebral vasospasm into two types: proximal-type vasospasm involving M1 and distal-type vasospasm not involving M1 and located at the distal part of the MCA (M2 and/or M3).

A large retrospective study comparing relative and absolute TCD velocity parameters for the detection of symptomatic MCA vasospasm demonstrated that absolute velocity thresholds were superior to relative velocity changes [10]. In this study, we defined the positive findings on TCCFI examination as a maximum flow velocity $>200 \mathrm{~cm} / \mathrm{s}$ or a mean flow velocity $>120$ $\mathrm{cm} / \mathrm{s}$ at M1. We defined group A as the patients who had positive findings on TCCFI examination and group $\mathrm{B}$ as the patients who had negative findings on TCCFI examination. Additionally, we investigated the flow velocity on TCCFI examination after the onset of delayed cerebral ischemia compared to before.

\section{Statistical Analysis}

For non-normally distributed continuous variables, the Mann-Whitney U testwas performed. To determine whether there was a difference in outcome between group A and group B, we defined a good outcome as a modified Rankin scale score of 1 and 2 and conducted a $\chi^{2}$ test. 
Table 2. Comparison of groups A and B

\begin{tabular}{lll}
\hline & Group A (\%) & Group B (\%) \\
\hline Transcranial color flow image & & \\
Positive increase in maximum blood flow velocity & $13(92.9)$ & 0 \\
Positive increase in mean blood flow velocity & $14(100)$ & 0 \\
Localization of vasospasm on angiography & $2(14.3)$ & 0 \\
MCA (including M1) & $4(28.6)$ & 0 \\
MCA (including M1) + ACA & $2(14.3)$ & 0 \\
MCA (including M1) + ICA & $2(14.3)$ & 0 \\
MCA (including M1) + ACA + ICA & $1(7.1)$ & 0 \\
MCA (including M1) + PCA & $1(7.1)$ & 0 \\
MCA (including M1) + ICA + PCA & $2(14.3)$ & 0 \\
MCA (including M1) + ACA + PCA & 0 & $2(33.3)$ \\
Distal part of the MCA (M2, M3) & 0 & $1(16.7)$ \\
Distal part of the MCA (M3) & 0 & $3(50.0)$ \\
Distal part of the MCA (M2, M3) + ACA & 0 & \\
\hline
\end{tabular}

ACA = Anterior cerebral artery; ICA = internal carotid artery; PCA = posterior cerebral artery.

\section{Results}

Twenty patients ( 9 men and 11 women) were included in this study. The mean age was 56 years (range 34-82). Fourteen patients had positive findings on TCCFI examination (group A), whereas the other 6 patients had negative findings on TCCFI examination (group $B$; table 1). The mean age of group A was significantly younger than that of group B ( $p<$ 0.01). The other variables of the clinical characteristics were not significantly different between group A and group B. All 14 patients of group A had angiographic vasospasm involving M1, whereas all 6 patients of group B had angiographic vasospasm localized at the distal part of the MCA (M2 and/or M3; table 2). All 20 patients were not hyperemic on SPECT measurement.

As to blood flow velocity at the initial TCCFI examination, both maximum and mean velocities were significantly lower in group B (maximum: $95.7 \mathrm{~cm} / \mathrm{s}$; mean: $56.2 \mathrm{~cm} / \mathrm{s}$ ) than in group A (maximum: $137.7 \mathrm{~cm} / \mathrm{s}$; mean: $89.6 \mathrm{~cm} / \mathrm{s}$ ). Comparing blood flow velocity on TCCFI before and after the onset of vasospasm, increases in the maximum and mean velocity were significantly smaller in group B than in group A (maximum: $18.2 \mathrm{~cm} / \mathrm{s}$ in group B vs. $94.0 \mathrm{~cm} / \mathrm{s}$ in group A; mean: $14.6 \mathrm{~cm} / \mathrm{s}$ in group B vs. $75.92 \mathrm{~cm} / \mathrm{s}$ in group A; fig. 1).

\section{Representative Cases}

A Representative Case of Group A (Case 1)

A 34-year-old male patient presented with SAH due to a ruptured basilar artery aneurysm. He developed delayed cerebral ischemia with aphasia and right hemiparesis on day 21 after SAH. A diffusion-weighted image showed high intensity spots in the left parietal lobe (fig. 2a). The TCCFI examination showed that the maximum and mean velocities at the left MCA were 237 and $186 \mathrm{~cm} / \mathrm{s}$, respectively. A left internal carotid angiography showed diffuse vasospasm involving the left M1 (fig. 2c, black arrows). Angiography of the left internal carotid on admission is also shown (fig. $2 \mathrm{~b}$ ).

Two Representative Cases of Group B (Cases 2 and 3)

Case 2. A 79-year-old male patient presented with SAH due to a ruptured anterior communicating artery aneurysm. He developed delayed cerebral ischemia with aphasia and distur- 


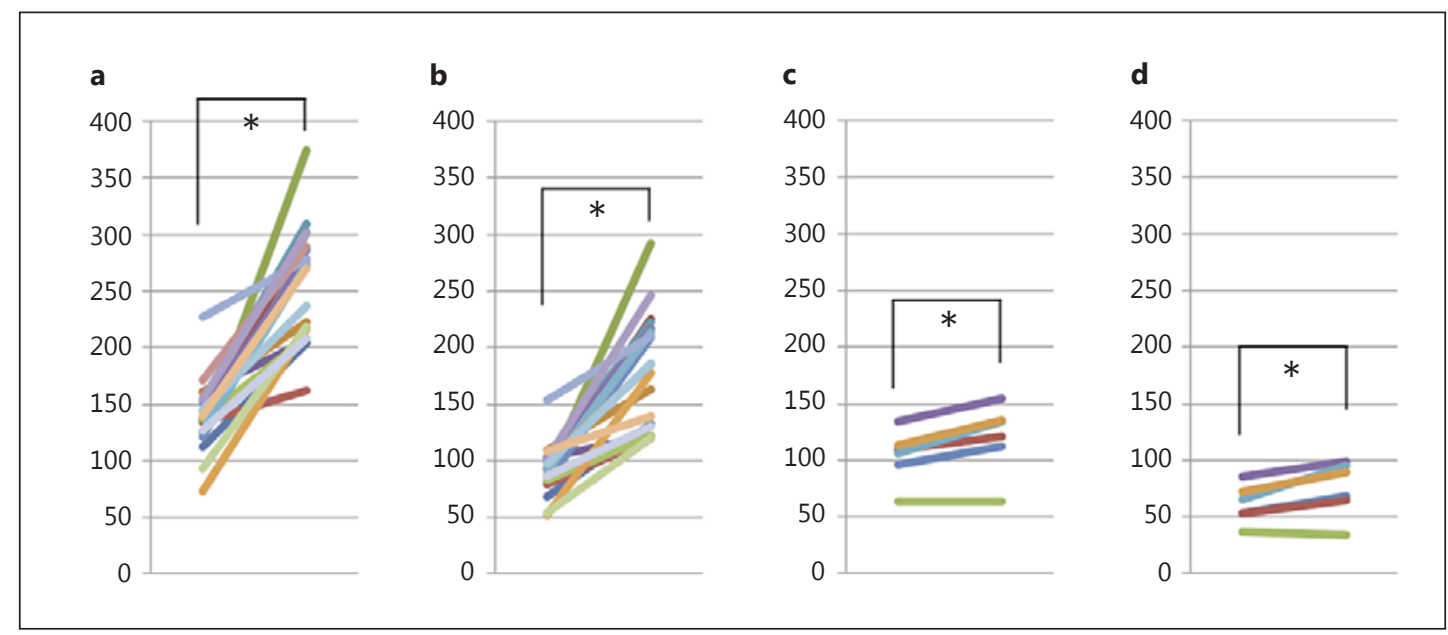

Fig. 1. Comparison of blood flow velocity $(\mathrm{cm} / \mathrm{s})$ on TCCFI before and after the onset of delayed cerebral ischemia. a Maximum velocity of group A. b Mean velocity of group A. c Maximum velocity of group B. d Mean velocity of group B. $*$ p $<0.01$.
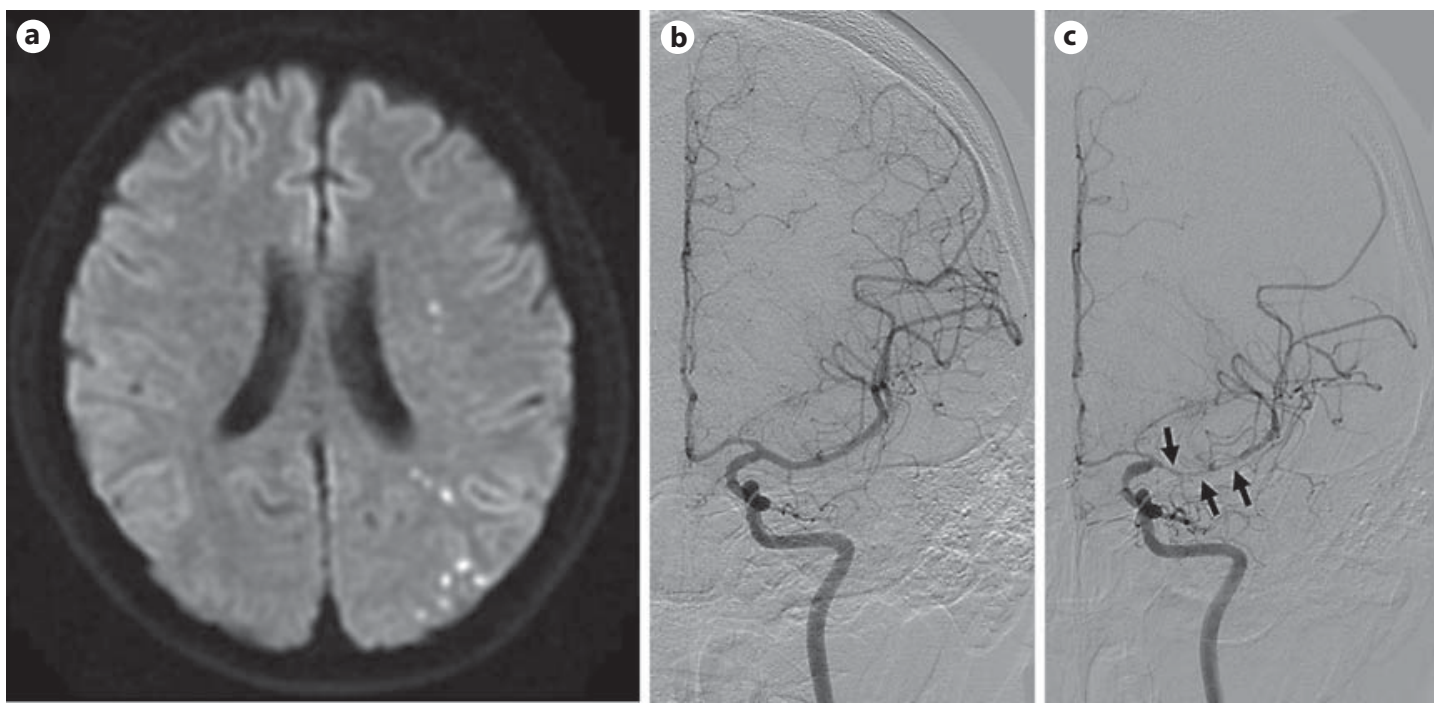

Fig. 2. Representative case of group A (case 1). a Diffusion-weighted image at the onset of delayed cerebral ischemia. b Left internal carotid angiography on admission. c Left internal carotid angiography showing diffuse vasospasm involving the left M1 (black arrows).

bance of consciousness on day 7 after SAH. A diffusion-weighted image showed multiple highintensity spots in the left hemisphere (fig. 3a). The TCCFI examination showed that the maximum and mean velocities at the left MCA were 135 and $89 \mathrm{~cm} / \mathrm{s}$, respectively. A left internal carotid angiography showed vasospasm localized at the distal part of the left MCA (fig. 3c, black arrows). Angiography of the left common carotid on admission is also shown (fig. 3b).

Case 3. A 73-year-old female patient presented with SAH due to a ruptured aneurysm at the bifurcation of a left internal carotid artery and a posterior communicating artery. She developed delayed cerebral ischemia with aphasia on day 10 after SAH. A diffusion-weighted image showed multiple high-intensity spots in the left hemisphere (fig. 4a). The TCCFI exam- 

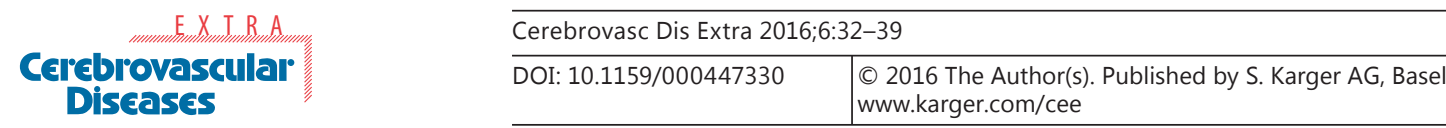

Kohama et al.: Difference in TCD Velocity and Patient Age between Proximal and Distal MCA Vasospasms after Aneurysmal SAH
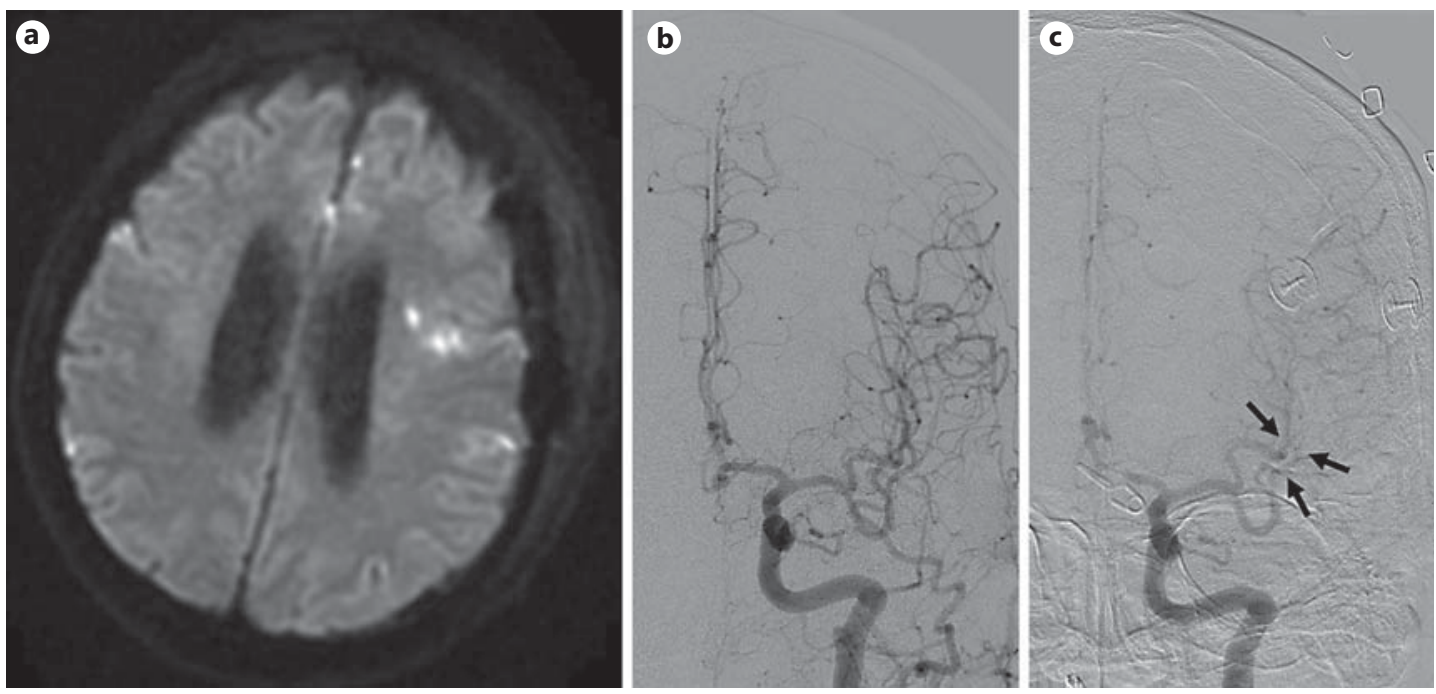

Fig. 3. Representative case of group B (case 2). a Diffusion-weighted image at the onset of delayed cerebral ischemia. b Left common carotid angiography on admission. c Left internal carotid angiography showing vasospasm localized at the distal part of the left MCA (black arrows).

ination showed that the maximum and mean velocities at the left MCA were 67 and $49 \mathrm{~cm} / \mathrm{s}$, respectively. A left internal carotid angiography showed vasospasm localized at the distal part of the left MCA (fig. 4c, black arrows). Angiography of the left internal carotid on admission is also shown (fig. 4b).

\section{Discussion}

TCD/TCCFI examination is reported to have limited ability to detect cerebral vasospasm after SAH $[6,11,12]$. The results of the present study showed that flow velocity at M1 did not increase to the values we defined as positive findings when the vasospasm was localized at the distal part of the MCA (M2 and/or M3). Therefore, TCCFI provides false-negative information when a vasospasm is localized at the distal part of the MCA.

The volume of blood flow $\left[V\left(\mathrm{~m}^{3} / \mathrm{s}\right)\right]$ is the multiplication of the surface of an artery $\left[S\left(\mathrm{~m}^{2}\right)\right]$ and the mean velocity of blood flow over the surface $[\bar{v}(\mathrm{~m} / \mathrm{s})]$ :

$$
V=\bar{v} \cdot S
$$

At the constant flow volume $(V)$, the velocity $(\bar{v})$ is inversely proportional to the surface of an artery (S). The diagnosis for cerebral vasospasm on TCD/TCCFI examination is based on this correlation, although the velocity measured by TCD/TCCFI $[v(\mathrm{~m} / \mathrm{s})]$ is not equal to $\bar{v}$. However, at the constant arterial surface (S), $\bar{v}$ (or $v$ ) does not increase, unless the flow volume $(V)$ increases. In patients with delayed cerebral ischemia, the flow volume $(V)$ does not increase because of the elevated resistance of peripheral arteries. In this study, SPECT showed that none of the cases were hyperemic. Under the condition that $V$ did not increase, the velocity of M1 (v) did not increase when M1 was not involved in the cerebral vasospasm, and the surface of M1 (S) was constant.

The distal type of vasospasm at the MCA in which TCCFI provided false negatives was seen in $6(30 \%)$ of 20 cases in the current study. The age of the 6 patients was significantly greater than that of the other 14 cases. Two pathophysiological reasons for this are considered. 


\section{Cerebrovascular \\ Diseases}

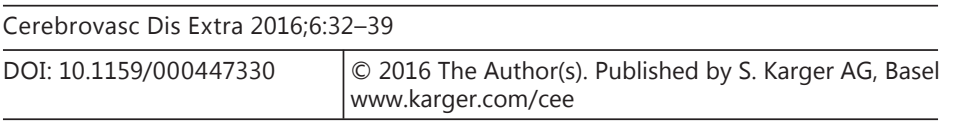

Kohama et al.: Difference in TCD Velocity and Patient Age between Proximal and Distal MCA Vasospasms after Aneurysmal SAH
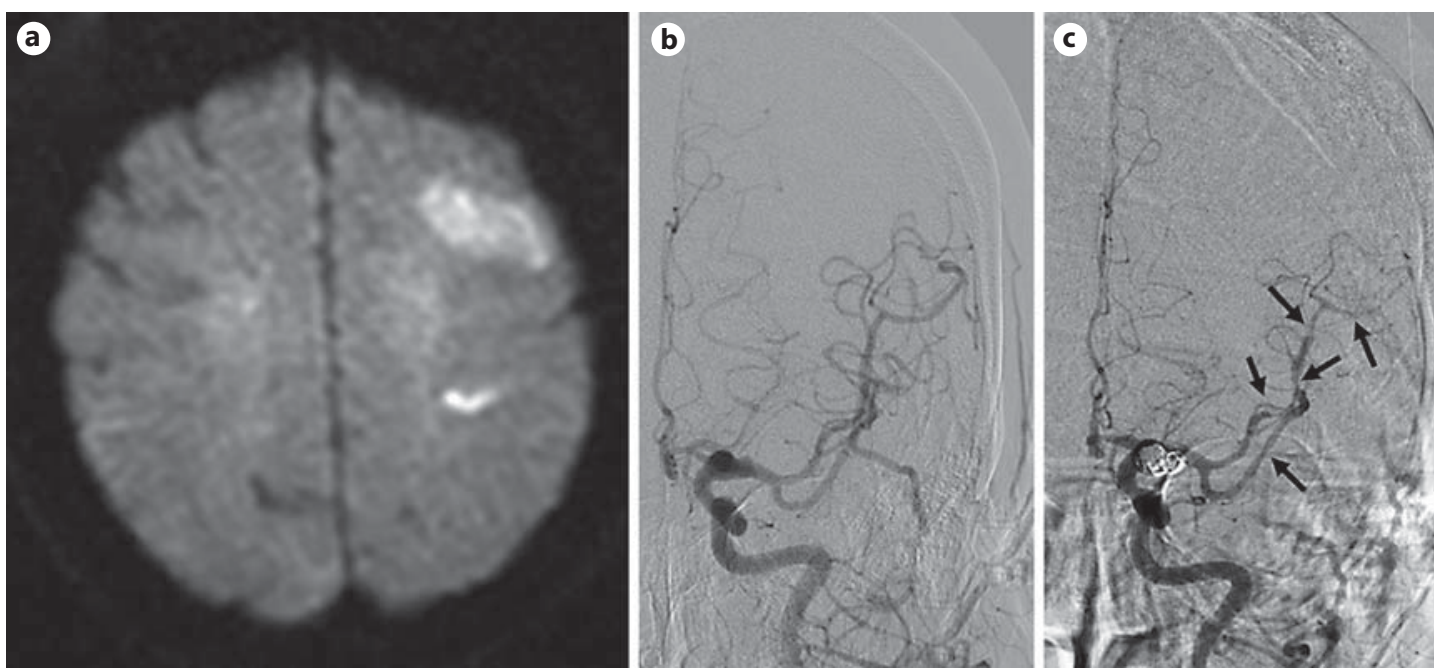

Fig. 4. Representative case of group B (case 3). a Diffusion-weighted image at the onset of delayed cerebral ischemia. b Left internal carotid angiography on admission. c Left internal carotid angiography showing vasospasm localized at the distal part of the left MCA (black arrows).

First, age-related atherosclerotic changes weaken vasoconstriction of the M1, thereby decreasing the contractility of the basal cerebral arteries. The second explanation is related to the reserve capacity of cerebral blood flow. Decreased cerebral blood flow and the absence of collateral flow in elderly patients narrows the margin between adequate cerebral blood flow and the ischemic threshold, making elderly patients susceptible to ischemic neurological deficits with even small changes in vessel caliber [10,13-15].

To improve the diagnosability of TCD/TCCFI for the distal type of vasospasm, we propose that a slight increase in flow velocity at the onset of vasospasm is important. In fact, the TCCFI examination at the onset of vasospasm showed a slight increase in flow velocity compared with the data at the initial TCCFI examination even in patients with distal-type vasospasm (fig. 1). These results suggest a slight decrease in M1 surface due to vasoconstriction which could not be detected in angiography. Given that flow velocity at the initial TCCFI examination was low and the increase in velocity at the onset of vasospasm was small, neither the maximum nor mean velocity at the onset of vasospasm reached the values we defined as a positive finding (maximum: $200 \mathrm{~cm} / \mathrm{s}$; mean: $120 \mathrm{~cm} / \mathrm{s}$ ). Therefore, in order to reduce false negatives, a slight increase in flow velocity on TCD/TCCFI examination should be recognized as positive for the diagnosis of distal-type vasospasm, especially in aged patients.

\section{Limitations}

The present study did not refer to the specificity or positive predictive value of TCD/ TCCFI because we selected patients who had true vasospasm in order to investigate the cause of false negatives on TCD/TCCFI. However, a previous review compensates for this limitation, as it reported that the specificity and positive predictive value of TCD/TCCFI were high [6]. It is important to understand such characteristics of TCD/TCCFI examination for diagnosing cerebral vasospasm after SAH.

The small size of the study population is also a limitation. Only 20 cases were included in this study, mainly due to the availability of angiography. Using magnetic resonance angiography instead of conventional angiography may allow for further investigation with a larger population to reinforce the findings of this initial study. 


\section{Conclusions}

TCD/TCCFI examination provided false-negative findings for the diagnosis of cerebral vasospasm localized at the distal part of the MCA because flow velocity at the horizontal part of the MCA did not increase to the level we defined as positive. A slight increase in flow velocity on TCD/TCCFI examination should be recognized as a positive finding for the distal type of vasospasm, especially in aged patients.

\section{Acknowledgement}

We thank Dr. Ayako Shimizu (Tohoku University Graduate School of Medicine, Sendai, Japan) for her technical support.

\section{Disclosure Statement}

The authors report no conflicts of interest.

\section{References}

1 van Gijn J, Kerr RS, Rinkel GJ: Subarachnoid haemorrhage. Lancet 2007;369:306-318.

-2 Charpentier C, Audibert G, Guillemin F, Civit T, Ducrocq X, Bracard S, Hepner H, Picard L, Laxenaire MC: Multivariate analysis of predictors of cerebral vasospasm occurrence after aneurysmal subarachnoid hemorrhage. Stroke 1999;30:1402-1408.

- 3 Rabinstein AA, Friedman JA, Weigand SD, McClelland RL, Fulgham JR, Manno EM, Atkinson J, Wijdicks EF: Predictors of cerebral infarction in aneurysmal subarachnoid hemorrhage. Stroke 2004;35:1862-1866.

-4 Suarez JI, Qureshi AI, Yahia AB, Parekh PD, Tamargo RJ, Williams MA, Ulatowski JA, Hanley DF, Razumovsky AY: Symptomatic vasospasm diagnosis after subarachnoid hemorrhage: evaluation of transcranial Doppler ultrasound and cerebral angiography as related to compromised vascular distribution. Crit Care Med 2002; 30:1348-1355

5 Aaslid R, Markwalder TM, Nornes H: Noninvasive transcranial Doppler ultrasound recording of flow velocity in basal cerebral arteries. J Neurosurg 1982;57:769-774.

-6 Lysakowski C, Walder B, Costanza MC, Tramer MR: Transcranial Doppler versus angiography in patients with vasospasm due to a ruptured cerebral aneurysm: a systematic review. Stroke 2001;32:2292-2298.

-7 Carrera E, Schmidt JM, Oddo M, Ostapkovich N, Claassen J, Rincon F, Seder D, Gordon E, Kurtz P, Lee K, Connolly ES, Badjatia N, Mayer SA: Transcranial Doppler ultrasound in the acute phase of aneurysmal subarachnoid hemorrhage. Cerebrovasc Dis 2009;27:579-584.

-8 Connolly ES Jr, Rabinstein AA, Carhuapoma JR, Derdeyn CP, Dion J, Higashida RT, Hoh BL, Kirkness CJ, Naidech AM, Ogilvy CS, Patel AB, Thompson BG, Vespa P; American Heart Association Stroke Council; Council on Cardiovascular Radiology and Intervention; Council on Cardiovascular Nursing; Council on Cardiovascular Surgery and Anesthesia; Council on Clinical Cardiology: Guidelines for the management of aneurysmal subarachnoid hemorrhage: a guideline for healthcare professionals from the American Heart Association/ American Stroke Association. Stroke 2012;43:1711-1737.

9 Fischer E: Lageabweichungen der vorderen Hirnarterie im Gefässbild. Zentralbl Neurochir 1938;3:300-312.

-10 Malhotra K, Conners JJ, Lee VH, Prabhakaran S: Relative changes in transcranial Doppler velocities are inferior to absolute thresholds in prediction of symptomatic vasospasm after subarachnoid hemorrhage. J Stroke Cerebrovasc Dis 2014;23:31-36.

-11 Carrera E, Schmidt JM, Oddo M, Fernandez L, Claassen J, Seder D, Lee K, Badjatia N, Connolly ES Jr, Mayer SA: Transcranial Doppler for predicting delayed cerebral ischemia after subarachnoid hemorrhage. Neurosurgery 2009;65:316-323.

-12 Findlay JM, Nisar J, Darsaut T: Cerebral vasospasm: a review. Can J Neurol Sci 2016;43:15-32.

$\checkmark 13$ Torbey MT, Hauser TK, Bhardwaj A, Williams MA, Ulatowski JA, Mirski MA, Razumovsky AY: Effect of age on cerebral blood flow velocity and incidence of vasospasm after aneurysmal subarachnoid hemorrhage. Stroke 2001;32:2005-2011.

-14 Boecher-Schwarz HG, Ungersboeck K, Ulrich P, Fries G, Wild A, Perneczky A: Transcranial Doppler diagnosis of cerebral vasospasm following subarachnoid haemorrhage: correlation and analysis of results in relation to the age of patients. Acta Neurochir (Wien) 1994;127:32-36.

15 Wachter D, Hans F, Kreitschmann-Andermahr I, Rohde V: Lower incidence of transcranial Doppler and symptomatic vasospasm after aneurysmal subarachnoid hemorrhage and aneurysm clipping in the elderly patient? Neurosurgery 2001;69:261-266. 